\title{
Lagrangian coherent structures in $n$-dimensional systems
}

\author{
Francois Lekien \\ Mechanical and Aerospace Engineering, Princeton University, Princeton, \\ New Jersey 08544 \\ Shawn C. Shadden ${ }^{\text {a) }}$ \\ Department of Bioengineering, Stanford University, Stanford, California 94305-5431 \\ Jerrold E. Marsden \\ Control and Dynamical Systems, California Institute of Technology, Pasadena, \\ California 91125
}

(Received 7 September 2006; accepted 23 April 2007; published online 13 June 2007)

\begin{abstract}
Numerical simulations and experimental observations reveal that unsteady fluid systems can be divided into regions of qualitatively different dynamics. The key to understanding transport and stirring is to identify the dynamic boundaries between these almost-invariant regions. Recently, ridges in finite-time Lyapunov exponent fields have been used to define such hyperbolic, almost material, Lagrangian coherent structures in two-dimensional systems. The objective of this paper is to develop and apply a similar theory in higher dimensional spaces. While the separatrix nature of these structures is their most important property, a necessary condition is their almost material nature. This property is addressed in this paper. These results are applied to a model of Rayleigh-Bénard convection based on a threedimensional extension of the model of Solomon and Gollub. (c) 2007 American Institute of Physics. [DOI: 10.1063/1.2740025]
\end{abstract}

\section{INTRODUCTION}

There is mounting computational and empirical evidence that hyperbolic structures strongly influence the transport geometry in both laminar and turbulent fluid systems. The role of hyperbolic structures in steady, periodic, and near-integrable planar systems has been well studied [Guckenheimer and Holmes (1986); Ottino (1989); Rom-Kedar et al. (1990); Rom-Kedar and Wiggins (1991)]. For these restricted classes of systems, hyperbolic, saddle-type fixed points exponentially stretch nearby trajectories, and the extended influence of these hyperbolic points can be understood in terms of their stable and unstable manifolds. For systems with aperiodic time dependence, characterizing the most important hyperbolic structures is not as straightforward. Nevertheless, the study of Lagrangian coherent structures ${ }^{1}$ (LCS) helps extend these constructs to aperiodic systems to allow a more precise characterization of the flow than traditional methods.

There have recently been a handful of methods proposed for locating LCS, which have mostly focused on analyzing planar systems. First, one can fix a portion of time and identify uniformly hyperbolic trajectories and then grow their associated finite-time invariant manifolds [Haller and Poje (1998); Mancho et al. (2004); Toner and Poje (2004)] to define LCS. This approach is similar to traditional methods for construction of stable and unstable manifolds [Homburg et al. (2003); Krauskopf et al. (2005)]. It consists in "straddling" the manifolds and iteratively constructing them. This method is attractive because it obtains a fairly explicit construction of the LCS. However, it is often difficult to compute LCS from this method in practical fluid applications due

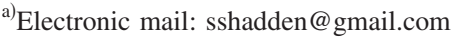

${ }^{1}$ Throughout this paper, LCS is used both for the singular and plural forms, where the appropriate abbreviation should be clear from context.
} 
to their nonuniqueness, the explicit reference to the equations of motion in the definition, and the conditions that must be satisfied to guarantee their existence. ${ }^{2}$ Moreover this approach is also difficult to extend to systems with dimension greater than 2 since it requires an explicit solution of the linearized dynamics inside the LCS. Such solutions are unavailable for LCS of dimensions higher than 1, and hence spaces with dimensions higher than 2 .

Alternatively, one can derive a measure of hyperbolic stretching and define LCS as the most hyperbolic structures. This approach is usually more robust than direct methods for systems with arbitrary time dependence. For planar systems, LCS are defined as curves that maximize some measure of hyperbolicity over a fixed time horizon. This "averaging" may result in LCS that are not exactly transport barriers. However, numerous applications have shown that the resulting distributed flux in a fixed horizon approach is very small and justifies its use as a means to define the LCS rigorously [Shadden et al. (2005)].

In Shadden et al. (2005), LCS are defined from finite-time Lyapunov exponent (FTLE) fields, an idea proposed by Haller $(2001,2002)$. The FTLE is a robust approximation of local hyperbolicity, the average rate of stretching about a single trajectory. Numerical simulations [Haller (2001); Lekien et al. (2005b); Green et al. (2007)], experimental results [Voth et al. (2002); Shadden et al. (2006)], and theoretical considerations [Shadden et al. (2005)] demonstrate that the FTLE field for planar systems reveals coherent lines that behave as separatrices in a wide variety of applications.

The ridge definition in Shadden et al. (2005) focused on the use of FTLE fields in defining LCS. Using FTLE is, however, not necessary and ridges can be computed from other measures of hyperbolicity, such as hyperbolicity time [Haller $(2000,2001)]$, finite strain [Jones and Winkler (2002)], or finite-size Lyapunov exponents (FSLE) [Koh and Legras (2002); Joseph and Legras (2002)].

The objective of this paper is to extend the definition and results in Shadden et al. (2005) to higher dimensional systems. In this case, we seek hypersurfaces of dimension 1 less than the phase space of the dynamical system. For periodic and near-integrable systems, there has been progress on computing invariant manifolds for systems with dimension greater than 2 [see Krauskopf et al. (2005) and Wysham and Meiss (2006)]. Relatively few results exist for computing invariant manifolds for aperiodic systems with dimension greater than 2, with Haller (2001) and Padberg (2005) being exceptions. The difficulties between computing LCS in two dimensions and three dimensions are that (1) it is no longer possible to obtain a global parameterization of the structure and (2) although the dimension increases by 1, the possible flow configurations increase by several. This second issue presents a more challenging concern for iterative methods, but both of these issues must be addressed in extending the results of Shadden et al. (2005).

In Sec. II we define LCS for $n$-dimensional dynamical systems and in Sec. III we derive some properties of these structures, including a bound to describe the Lagrangian behavior. To illustrate the concepts developed, we compute the LCS for a model of unsteady, three-dimensional Rayleigh-Bénard convection in Sec. IV.

\section{DEFINITIONS}

We study the dynamical system

$$
\dot{\mathbf{x}}(t)=\mathbf{v}(\mathbf{x}(t), t),
$$

where $\mathbf{x} \in \mathcal{D} \subset \mathbb{R}^{n}$ together with a piecewise smooth boundary, and with the following assumptions:

$A 1$. The velocity field is at least $C^{0}$ in time and $C^{3}$ in space.

A2. The domain $\mathcal{D}$ is compact.

\footnotetext{
${ }^{2}$ Straddling requires the existence of uniformly hyperbolic trajectories, which are difficult to locate in most practical applications because hyperbolic trajectories regularly become instantaneously nonhyperbolic due to short-term anomalies.
} 
The first assumption is required to allow for the calculus needed to prove the analytic results of this paper. Although this condition can be ensured by proper interpolation when working with data, it is typically not needed for practical purposes or numerical stability. For example, if $\mathbf{v}$ is specified on a Cartesian mesh, Lekien and Marsden (2005) provide a $C^{1}$ tricubic interpolation, which is typically sufficient. Unstructured data can be interpolated by normal modes for complex boundaries [Lekien et al. (2004)]. Additionally, nearly all examples of practical importance must be handled numerically, so there is no loss of generality by assuming that the domain is closed and bounded, and hence compact.

The flow map is denoted by $\phi_{t}^{T}: \mathbf{x}(t) \mapsto \mathbf{x}(t+T)$, which is simply the solution to Eq. (1) written as a mapping whose input is the initial location of a point at time $t$ and whose output is the position at time $t+T$. We begin by recalling two important properties of the flow map that we will later exploit.

Theorem 2.1: For each fixed $t_{0}>0$ and $T_{0}>0$, there is a constant $K>0$ such that

$$
\left\|\frac{\mathrm{d} \phi_{t}^{T}(\mathbf{x})}{\mathrm{d} \mathbf{x}}\right\| \leqslant \mathrm{e}^{K|T|},
$$

for all $\mathbf{x} \in \mathcal{D}$, all $t$ satisfying $0 \leqslant|t| \leqslant t_{0}$, and all $T$ satisfying $0 \leqslant|T| \leqslant T_{0}$.

This theorem results from the fact that we have taken $\mathbf{v}$ to be $C^{3}$ in space. Gronwall's inequality is typically used in standard textbooks to prove this result [Hirsh and Smale (1974)].

Also note that since the flow is invertible, the linearization of the flow map is nonsingular, and hence

$$
\left\|\frac{\mathrm{d} \phi_{t}^{T}(\mathbf{x})}{\mathrm{d} \mathbf{x}}\right\|>0
$$

for arbitrary $T$.

\section{A. Finite-time Lyapunov exponents}

The growth of an infinitesimal perturbation $\boldsymbol{\delta}(t) \neq \mathbf{0}$ to a point $\mathbf{x}(t)$ obeys

$$
0<\left\|\frac{\mathrm{d} \phi_{t}^{T}(\mathbf{x})}{\mathrm{d} \mathbf{x}} \boldsymbol{\delta}(t)\right\|^{2}=\left\langle\frac{\mathrm{d} \phi_{t}^{T}(\mathbf{x})}{\mathrm{d} \mathbf{x}} \boldsymbol{\delta}(t), \frac{\mathrm{d} \phi_{t}^{T}(\mathbf{x})}{\mathrm{d} \mathbf{x}} \boldsymbol{\delta}(t)\right\rangle=\left\langle\boldsymbol{\delta}(t), \frac{\mathrm{d} \phi_{t}^{T}(\mathbf{x})^{*}}{\mathrm{~d} \mathbf{x}} \frac{\mathrm{d} \phi_{t}^{T}(\mathbf{x})}{\mathrm{d} \mathbf{x}} \boldsymbol{\delta}(t)\right\rangle=\|\boldsymbol{\delta}(t+T)\|^{2},
$$

where the symbol * denotes the adjoint (transpose). The strict inequality in Eq. (3) is a consequence of Eq. (2). Equation (3) highlights the importance of the finite-time deformation tensor ${ }^{3}$

$$
\Delta(\mathbf{x}, t ; T)=\frac{\mathrm{d} \phi_{t}^{T}(\mathbf{x})^{*}}{\mathrm{~d} \mathbf{x}} \frac{\mathrm{d} \phi_{t}^{T}(\mathbf{x})}{\mathrm{d} \mathbf{x}}
$$

in the study of attraction and separation in fluids. We may regard $\mathbf{\Delta}(\mathbf{x}, t ; T)$ as a symmetric, positive definite, linear operator on $\mathbb{R}^{n}$. As a result, $\Delta(\mathbf{x}, t ; T)$ has $n$ real, positive eigenvalues and we define the FTLE as

$$
\sigma(\mathbf{x}, t ; T) \triangleq \frac{1}{|T|} \ln \sqrt{\lambda_{\max }(\Delta(\mathbf{x}, t ; T))},
$$

where $\lambda_{\max }(\Delta(\mathbf{x}, t ; T))$ denotes the maximum eigenvalue of $\boldsymbol{\Delta}(\mathbf{x}, t ; T)$.

It is easily shown that the norm of a perturbation $\boldsymbol{\delta}(t)$ to a point $\mathbf{x}(t)$ evolves as

\footnotetext{
${ }^{3}$ Also called the (finite-time) Cauchy-Green tensor.
} 


$$
\|\boldsymbol{\delta}(t+T)\| \leqslant \mathrm{e}^{\sigma(\mathbf{x}, t ; T)|T|}\|\boldsymbol{\delta}(t)\|,
$$

where strict equality holds if $\boldsymbol{\delta}(t)$ is chosen in the eigenvector direction of $\lambda_{\max }(\boldsymbol{\Delta}(\mathbf{x}, t ; T))$. Furthermore, most $\boldsymbol{\delta}(t)$ will typically tend to this most unstable eigenvalue direction over time. Note that the integration time $T$ can be positive or negative, corresponding to the forward-time and backward-time FTLE. In the next section, we build LCS as hypersurfaces that "locally maximize" the FTLE field. The type of LCS obtained depends on the sign of the integration time $T$; attracting LCS are obtained from negative $T$ and repelling LCS are obtained from positive $T$.

When the integration time $T$ is too short, the FTLE only reveals time-varying features in the velocity gradient and therefore is not indicative of particle transport when the system is unsteady. When $T$ is too large, the FTLE can contain averaged information for several different dynamic regimes of the flow if the system has general aperiodic time dependence. Consideration for the time scales of the flow and numerical experimentation is usually sufficient to determine an appropriate integration time [Lekien and Leonard (2004)]. For most practical purposes, experience dictates that LCS are not sensitive to variations of $T$ and become better resolved as $|T|$ increases [Coulliette et al. (to be published)].

A criterion similar to the FTLE is the FSLE, which consists of measuring how much time it takes for a sphere of radius $r_{0}$ about a trajectory to reach a distance $r_{f}>r_{0}$. The inverse of this time, weighted by $\ln \left(r_{f} / r_{0}\right)$, tends to the FTLE as $r_{f} \rightarrow 0$ (while keeping $r_{0}<r_{f}$ ). In this case, the effective integration time $T$ is not constant; it depends on the choices for $r_{0}, r_{f}$, and the actual value of the FTLE. One can think of this method as a way to change the integration time $T$ in the definition of the FTLE based on local properties. For systems with marked regimes such as seasonal geophysical events, the integration time $T$ can be determined based on the time scale of the event to observe [Lekien and Leonard (2004)]. For systems where inhomogeneous time scales govern the phenomena of interest, FSLE is sometimes more appropriate by providing a framework where the integration time $T$ adapts to the local stretching strength [Koh and Legras (2002)].

Whether the hyperbolicity measure $\sigma$ is defined by the FTLE or FSLE, it is important to differentiate trajectories from neighboring points in the grid used to discretize the computation of $\sigma$ as to not ignore lines of high stretching between grid points. Additionally, for the results listed herein to carry over to other criteria the only requirement is that $\sigma$ must be transported in some manner by the flow, as in the relationship given by Corollary 3.1. In this paper, we consider the FTLE; hence this condition is realized by the $\mathcal{O}(1 /|T|)$ term in Corollary 3.1. Using FSLE, one follows the same development with $\mathcal{O}\left(\left|r_{0} / r_{f}\right|,\left|r_{0}\right|\right)$.

\section{B. Lagrangian coherent structures}

Observations of FTLE fields for time-dependent fluid systems reveal the presence of organized regions of high relative FTLE values [Pierrehumbert (1991); Pierrehumbert and Yang (1993); Haller (2001, 2002); Inanc et al. (2005); Lekien et al. (2005b); Arratia et al. (2005); Shadden et al. $(2005,2006)]$. Suppose that the fluid is experiencing qualitatively different dynamics in two almost-invariant regions of the flow. In each of these regions, we expect a coherent motion of the fluid (such as in an eddy) and the eigenvalues of $\boldsymbol{\Delta}(\mathbf{x}, t ; T)$ in that region will be close to 1 , an indication that the fate of nearby particles is similar inside the subregion.

At the boundary of two regions of qualitatively different dynamics (e.g., two eddies with different vorticities), perturbations of the initial conditions can cause particles to move in one or the another region. This creates a higher eigenvalue in a direction transverse to the boundary. These boundaries or separatrices will be defined as LCS. The definition that we introduce below [the extension to $n$ dimensions of the definition in Shadden et al. (2005)] captures this aspect by requiring that one eigenvalue of $\boldsymbol{\Delta}(\mathbf{x}, t ; T)$ be greater than 1 while all the others have modulus less than 1 . We exclude cases where, along the boundary, we have multiple eigenvalues larger than 1. These cases correspond to hyperbolic structures that are not codimension 1. As a result, they do not qualify as hypersurfaces that divide regions, cf. Fig. 3 of Haller (2001). Notice that this creates a nontrivial difference between two-dimensional systems and higher dimensional systems. In two dimensions, excluding sources and sinks, there are only one-dimensional hyperbolic structures. In 
higher dimensions, we need to refine our conditions on the spectrum of $\boldsymbol{\Delta}(\mathbf{x}, t ; T)$ to exclude hyperbolic structures of codimension higher than 1 . This is motivated by our desire to define true codimension 1 boundaries. Nevertheless, chaotic dynamics may occur around lower level structures and the LCS in higher dimensional system may no longer be the only source of chaotic advection.

The other main difference with the two-dimensional theory presented in Shadden et al. (2005) is the fact that a global parametrization the LCS cannot be assumed. In two dimensions, the LCS can be represented by an explicit parametrization $\mathbf{p}(s)$ where $s \in] a, b[\subset \mathbb{R}$. In higher dimensions, such a global parametrization is usually nonexistent. We define the LCS as a manifold, a hypersurface that can locally be represented as the graph of a continuous function. This allows for a more general theory but necessitates a new formalism to extend the results in Shadden et al. (2005).

Definition 2.1: Consider a positive integration time $T$ with $\mathbf{\Delta}(\mathbf{x}, t ; T)$ and $\sigma(\mathbf{x}, t ; T)$ defined as above. A repelling $L C S$ is a condimension 1, orientable, differentiable manifold $\mathcal{M} \subset \mathcal{D} \subset \mathbb{R}^{n}$ satisfying the following conditions for each $\mathbf{x} \in \mathcal{M}$ :

LCS1. $\mathbf{\Delta}(\mathbf{x}, t ; T)$ has $n-1$ eigenvalues less than 1 and one eigenvalue greater than 1 .

LCS2. The unit normal vector $\hat{\mathbf{n}}(\mathbf{x})$ to the manifold $\mathcal{M}$ is orthogonal to $\nabla \sigma(\mathbf{x}, t ; T)$.

LCS3. Let $\Sigma$ be the second derivative of $\sigma(\mathbf{x} ; t ; T)$, thought of as a bilinear form evaluated at the point $\mathbf{x}$. We require that

$$
\Sigma(\hat{\mathbf{n}}, \hat{\mathbf{n}})<0,
$$

and for all unit vectors $\hat{\mathbf{u}}$ such that $|\langle\hat{\mathbf{u}}, \hat{\mathbf{n}}\rangle| \neq 1$,

$$
\boldsymbol{\Sigma}(\hat{\mathbf{n}}, \hat{\mathbf{n}})<\boldsymbol{\Sigma}(\hat{\mathbf{u}}, \hat{\mathbf{u}}) .
$$

We require that $\mathcal{M}$ be orientable to guarantee the existence and uniqueness of a continuous normal unit vector on $\mathcal{M}$. In general, $\mathcal{M}$ is orientable if there is a nonvanishing volume form on $\mathcal{M}$. In this paper, we will represent $\mathcal{M}$ as the level set of a $C^{2}$ function (cf. Definition 3.1 below) and use the gradient of the function to provide a well-defined normal.

To define attracting LCS, we consider the system with the flow reversed in time, that is, the backward-time flow $\phi_{t}^{-T}(\mathbf{x}): \mathbf{x}(t) \mapsto \mathbf{x}(t-T)$ is used to compute the FTLE field at time $t$. Attracting LCS will appears as repelling LCS when the flow is reversed in time. The motivation for this is that attracting LCS can be defined and computed by only slight modification of the definition or computational method used to obtain repelling LCS.

Definition 2.2: An attracting LCS is a repelling LCS of the backward-time flow (i.e., negative integration time $T$ ).

We next discuss the smoothness of the LCS. In general, the smoothness of the FTLE field or LCS is directly related to the smoothness of the vector field. As shown below, assumption A1 allows the LCS to be sufficiently well defined.

Lemma 2.1: The FTLE $\sigma(\mathbf{x}, t ; T)$ is $C^{3}$ in an open neighborhood $\mathcal{G}$ of the LCS.

Proof: Assumption A1 guarantees that the entries of the matrix $\boldsymbol{\Delta}(\mathbf{x}, t ; T)$ are $C^{3}$. This does not necessarily imply that $\lambda_{\max }$ is $C^{3}$ since the maximum value can switch from one eigenvalue to another in a nonsmooth manner. However, since the eigenvalues vary continuously, we are guaranteed that LCS1 holds in an open neighborhood $\mathcal{G}$ of the LCS. Inside $\mathcal{G}, \lambda_{\max }$ is outside the unit circle and all other eigenvalues are inside the unit circle. Therefore, $\lambda_{\max }$ does not undergo a switch, and thus [see, for instance, Kato (1980) and Dieci and Eirola (1999)], is $C^{3}$, making $\sigma(\mathbf{x}, t ; T)$ a $C^{3}$ function of $\mathbf{x}$.

Corollary 2.1: The normal vector $\hat{\mathbf{n}}$ to the LCS is $C^{1}$.

Proof: From Lemma 2.1, $\Sigma$ varies in a $C^{1}$ fashion. Since $\Sigma$ is $C^{1}$ and the smallest eigenvalue of $\Sigma$ is isolated, by LCS3, it follows that the associated eigenvector is $C^{1}$, as is well known [see, for instance, Kato (1980) or Dieci and Eirola (1999)]. This eigenvector (when normalized) corresponds to $\hat{\mathbf{n}}$ by LCS3. 


\section{FLUX}

For convenience, the following corollary from Shadden et al. (2005) is restated:

Corollary 3.1: The variation of the FTLE field satisfies

$$
\frac{\partial \nabla \sigma(\mathbf{x}, t ; T)}{\partial t}=-J^{*} \nabla \sigma(\mathbf{x}, t ; T)-\mathbf{\Sigma} \mathbf{v}+\mathcal{O}(1 /|T|),
$$

where $J$ is the spatial derivative (Jacobian matrix) of the velocity field $\mathbf{v}$.

Above, we use a classical abuse of notation and view $\Sigma$ as a linear operator (using the Riesz representation theorem). That is, we define $\Sigma \boldsymbol{\delta}$ to be the unique vector $\boldsymbol{\epsilon}$ such that $(\mathbf{y}, \Sigma \boldsymbol{\delta})$ $=\langle\mathbf{y}, \boldsymbol{\epsilon}\rangle$ for all $\mathbf{y} \in \mathbb{R}^{n}$. Corollary 3.1 will be used later in proving the Lagrangian properties of the LCS; this result is independent of the dimension of the system and the derivation in Shadden et al. (2005) of Eq. (5) above carries over to the $n$-dimensional case without modification.

\section{A. Representation}

Suppose that we are given a FTLE field $\sigma(\mathbf{x}, t ; T)$ for $t \in\left[t_{1}, t_{2}\right]$ of system (1) that admits a LCS in the sense of Definition 2.1 or 2.2. We define the scalar function $L$ of space and time as follows:

Definition 3.1: For all $t$, let $L(\mathbf{x}, t)$ be the function of $\mathbf{x} \in \mathcal{D}$ defined by the following conditions:

1. $|L(\mathbf{x}, t)|=\left\|\mathbf{x}-\mathbf{x}_{q}\right\|$, where $\mathbf{x}_{a}$ is a point on the LCS closest to point $\mathbf{x}$.

2. $L(\mathbf{x}, t)\left\langle\mathbf{x}-\mathbf{x}_{q}, \hat{\mathbf{n}}\left(\mathbf{x}_{q}\right)\right\rangle \geqslant 0$, where $\hat{\mathbf{n}}\left(\mathbf{x}_{q}\right)$ is the unit normal to the LCS at point $\mathbf{x}_{q}$.

The function $L(\mathbf{x}, t)$ gives the "signed distance" from $\mathbf{x}$ to the nearest point on the LCS. That is, the first condition for $L$ specifies the magnitude of the function as the distance between the points, and the second determines the sign of $L$. In a neighborhood of the LCS, points on one side of the LCS have a positive value of $L$ and a negative value on the other side. By definition of $L$, the LCS is given by the zero set $L=0$. This function is never computed in practical applications and is used in this work as a theoretical tool to derive the properties of the LCS. As shown in the next section, $\nabla L$ can be viewed as an extension of the normal vector $\hat{\mathbf{n}}$ away from the LCS.

\section{B. Properties}

Our objective is to analyze the Lagrangian properties of the LCS by analyzing the function $L$ defined above. For this purpose, the first and second derivatives of $L$ are needed. We need to start by showing that $L$ is $C^{2}$ near the LCS. Clearly $L$ is continuous everywhere. However, there may exist points in the domain that have multiple possible values for $\mathbf{x}_{q}$; hence $L$ might not be $C^{1}$ everywhere. However, we can prove that we can always find an open set that contains the LCS and excludes any of these points. Furthermore, we show that the second derivative of $L$ is continuous over this set.

Theorem 3.1: Let $\mathcal{B} \subset \mathcal{D}$ be the set of points with nonunique $\mathbf{x}_{q}$. The LCS and the closure of $\mathcal{B}$ are disjoint.

Proof: The critical ingredient in this proof is the fact that the curvature of the LCS must remain finite, and thus, close enough to the LCS, each point has a unique closest point on the LCS. The only prerequisite for this theorem is, therefore, the smoothness of the LCS, as given by Corollary 2.1. To prove the result, we will show that $\overline{\mathcal{B}}$ (i.e., the closure of $\mathcal{B}$ ) must be at a finite distance from the LCS by contradiction; if $\overline{\mathcal{B}} \cap \mathcal{M} \neq \varnothing$, then the curvature of the LCS would become infinite.

Consider an arbitrary point $\mathbf{p} \in \overline{\mathcal{B}} \cap \mathcal{M}$. In this case, $\mathbf{p}$ is in the closure of $\mathcal{B}$, so there exists at least one sequence $\mathbf{x}_{i} \in \mathcal{B}$ such that $\mathbf{x}_{i} \rightarrow \mathbf{p} \in \mathcal{M}$. By definition of $\mathcal{B}$, for each $\mathbf{x}_{i}$, there exists at least two separate points $\mathbf{x}_{i}^{1}$ and $\mathbf{x}_{i}^{2}$ on $\mathcal{M}$ that are equidistant from $\mathbf{x}_{i}$, and every other point on the LCS is located at the same distance from $\mathbf{x}_{i}$ as these points or farther. 


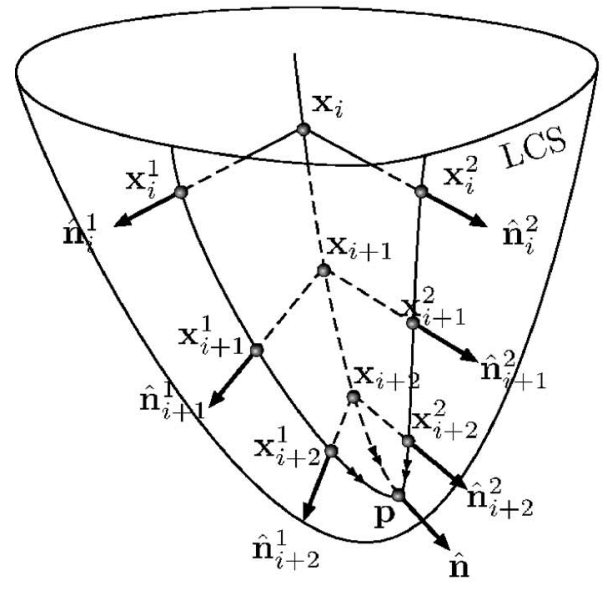

(a) Setting for the proof of Theorem 3.1

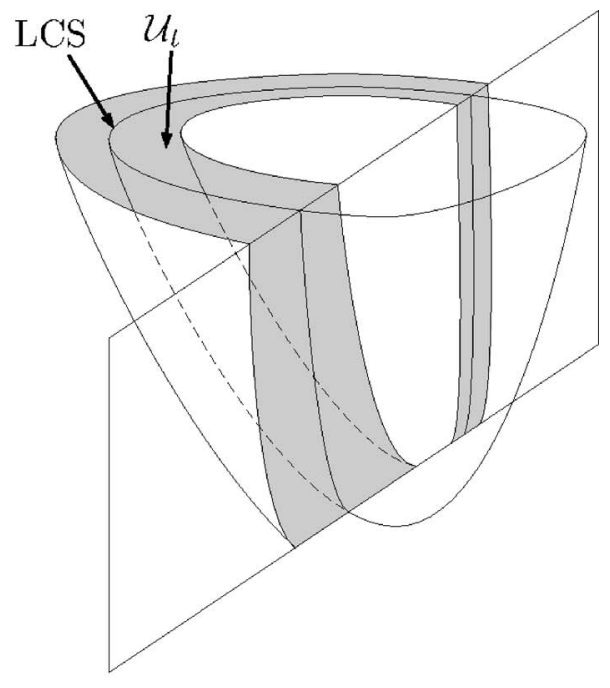

(b) An open set $\mathcal{U}_{t}$ enclosing the LCS, where $L$ is $C^{2}$

FIG. 1. For any LCS, there exists an open cover that excludes points of discontinuity of $L$.

Notice that $\left\|\mathbf{x}_{i}-\mathbf{p}\right\| \rightarrow 0$ as $i \rightarrow \infty$ and $\left\|\mathbf{x}_{i}^{1}-\mathbf{x}_{i}\right\|=\left\|\mathbf{x}_{i}^{2}-\mathbf{x}_{i}\right\| \leqslant\left\|\mathbf{p}-\mathbf{x}_{i}\right\|$ so we must have

$$
\begin{gathered}
\mathbf{x}_{i}^{1} \rightarrow \mathbf{p}, \\
\mathbf{x}_{i \rightarrow \infty}^{2} \rightarrow \mathbf{p}, \\
\mathbf{x}_{i}^{1} \neq \mathbf{x}_{i}^{2} \quad \text { for all } i .
\end{gathered}
$$

Since the LCS is a $C^{2}$ manifold, there exists an open set about point $\mathbf{p}, \mathcal{O}_{\mathbf{p}} \subset \mathcal{M}$, where the LCS can be described by a $C^{2}$ diffeomorphism, $h: A \subset \mathbb{R}^{n-1} \rightarrow \mathcal{O}_{\mathbf{p}} \subset \mathcal{M}$ (i.e., a local chart of the manifold containing the point $\mathbf{p})$. We will denote by $\mathbf{s}=\left(s_{1}, s_{2}, \ldots, s_{n-1}\right)$ the coordinates in the open set of $\mathbb{R}^{n-1}$. We can assume that the points $\mathbf{x}_{i}, \mathbf{x}_{i}^{1}$, and $\mathbf{x}_{i}^{2}$ are all contained in $\mathcal{O}_{\mathbf{p}}$ since the three sequences converge toward $\mathbf{p}$.

The mapping $h$ is a bijection; therefore for any $i$, there are unique $\mathbf{s}_{i}^{1}$ and $\mathbf{s}_{i}^{2}$ such that $h\left(\mathbf{s}_{i}^{1}\right)=\mathbf{x}_{i}^{1}$ and $h\left(\mathbf{s}_{i}^{2}\right)=\mathbf{x}_{i}^{2}$. Similarly, we denote by $\mathbf{s}_{\mathbf{p}}$ the unique point of $A$ such that $h\left(\mathbf{s}_{\mathbf{p}}\right)=\mathbf{p}$.

Notice that the largest principal curvature at point $\mathbf{p}$ equals the largest singular value of

$$
\left.\frac{\partial h^{-1} \hat{\mathbf{n}}(h(\mathbf{s}))}{\partial \mathbf{s}}\right|_{\mathbf{s}_{\mathbf{p}}} .
$$

Therefore, Corollary 2.1 implies that

$$
\left\|\frac{\partial \hat{\mathbf{n}}\left(h\left(\mathbf{s}_{\mathbf{p}}\right)\right)}{\partial \mathbf{s}} \delta \mathbf{s}\right\|<\infty,
$$

for any $\delta \mathbf{s}$. The converging sequences of Eq. (6) in $\mathcal{O}_{\mathbf{p}}$ allow us to express the derivative above as the limit of the difference between the normal vectors at points $\mathbf{x}_{i}^{1}$ and $\mathbf{x}_{i}^{2}$. Let us denote by $\hat{\mathbf{n}}_{i}^{1}$ and $\hat{\mathbf{n}}_{i}^{2}$ the unit vectors normal to the LCS at, respectively, $\mathbf{x}_{i}^{1}$ and $\mathbf{x}_{i}^{2}$; cf. Fig. 1 for the geometric interpretation of this setting for $n=3$. Since Eq. (7) holds for all $\delta \mathbf{s}$, we can choose 


$$
\delta \mathbf{s}=\lim _{i \rightarrow \infty} \frac{\mathbf{s}_{i}^{2}-\mathbf{s}_{i}^{1}}{\left\|\mathbf{s}_{i}^{2}-\mathbf{s}_{i}^{1}\right\|}
$$

From Eq. (7), we get the curvature

$$
\kappa \triangleq \lim _{i \rightarrow \infty} \frac{\left\|\hat{\mathbf{n}}_{i}^{2}-\hat{\mathbf{n}}_{i}^{1}\right\|}{\left\|\mathbf{s}_{i}^{2}-\mathbf{s}_{i}^{1}\right\|}<\infty .
$$
diction.

We will compute the limit in Eq. (8) and show that it goes unbounded, providing the contra-

Notice that points $\mathbf{x}_{i}^{1}$ and $\mathbf{x}_{i}^{2}$ are the points on the LCS that are the closest to $\mathbf{x}_{i}$; hence the vectors $\mathbf{x}_{i}^{1}-\mathbf{x}_{i}$ and $\mathbf{x}_{i}^{2}-\mathbf{x}_{i}$ must be aligned with, respectively, $\hat{\mathbf{n}}_{i}^{1}$ and $\hat{\mathbf{n}}_{i}^{2}$. Therefore the difference between the normal vectors can be written as

$$
\left\|\hat{\mathbf{n}}_{i}^{2}-\hat{\mathbf{n}}_{i}^{1}\right\|=\left\|\frac{\mathbf{x}_{i}^{2}-\mathbf{x}_{i}}{\left\|\mathbf{x}_{i}^{2}-\mathbf{x}_{i}\right\|}-\frac{\mathbf{x}_{i}^{1}-\mathbf{x}_{i}}{\left\|\mathbf{x}_{i}^{1}-\mathbf{x}_{i}\right\|}\right\|=\frac{\left\|\mathbf{x}_{i}^{2}-\mathbf{x}_{i}^{1}\right\|}{\left\|\mathbf{x}_{i}^{2}-\mathbf{x}_{i}\right\|},
$$

where we have used the fact that $\left\|\mathbf{x}_{i}^{1}-\mathbf{x}_{i}\right\|=\left\|\mathbf{x}_{i}^{2}-\mathbf{x}_{i}\right\|$. We also have

$$
\left\|\left.\frac{\partial h(\mathbf{s})}{\partial \mathbf{s}}\right|_{\mathbf{s}_{\mathbf{p}}} \delta \mathbf{s}\right\|=\lim _{i \rightarrow \infty} \frac{\left\|\mathbf{x}_{i}^{2}-\mathbf{x}_{i}^{1}\right\|}{\left\|\mathbf{s}_{i}^{2}-\mathbf{s}_{i}^{1}\right\|}=D_{\mathbf{p}}>0,
$$

because $h(\mathbf{s})$ is a $C^{1}$ diffeomorphism, and hence $\|\partial h(\mathbf{s}) / \partial \mathbf{s}\| \neq 0$ on the LCS and so must remain positive in a neighborhood of $\mathbf{s}=s_{\mathbf{p}}$ by continuity. As a result, there is an $i^{*}$ such that for all $i>i^{*}$,

$$
\frac{\left\|\mathbf{x}_{i}^{2}-\mathbf{x}_{i}^{1}\right\|}{\left\|\mathbf{s}_{i}^{2}-\mathbf{s}_{i}^{1}\right\|} \geqslant \frac{D_{\mathbf{p}}}{2}>0
$$

by definition of the limit.

Equations (9) and (10) give

$$
\kappa=\lim _{i \rightarrow \infty} \frac{1}{\left\|\mathbf{x}_{i}^{2}-\mathbf{x}_{i}\right\|} \frac{\left\|\mathbf{x}_{i}^{2}-\mathbf{x}_{i}^{1}\right\|}{\left\|\mathbf{s}_{i}^{2}-\mathbf{s}_{i}^{1}\right\|} \geqslant \frac{D_{\mathbf{p}}}{2} \lim _{i \rightarrow \infty} \frac{1}{\left\|\mathbf{x}_{i}^{2}-\mathbf{x}_{i}\right\|}=+\infty,
$$

which contradicts Eq. (8).

Lemma 2.1 states that $\sigma(\mathbf{x}, t ; T)$ is $C^{3}$ on an open set $\mathcal{G}$ containing the LCS. Theorem 3.1 shows that $\overline{\mathcal{B}}$, the closure of the set of points with nonunique $\mathbf{x}_{q}$, does not intersect the LCS. It is therefore natural to combine these two results and define

$$
\mathcal{U}_{t}=\{\mathcal{D} \backslash \overline{\mathcal{B}}\} \cap \mathcal{G}
$$

The resulting set $\mathcal{U}_{t}$ is an open set containing the LCS since both $\mathcal{G}$ and $\mathcal{D} \backslash \overline{\mathcal{B}}$ contain the LCS. Inside $\mathcal{U}_{t}$, the field $\sigma(\mathbf{x}, t ; T)$ is $C^{3}$ and each point has a unique $\mathbf{x}_{q}$. We next use this to prove the smoothness of $L$ inside this open cover.

Theorem 3.2: $L(x, t)$ is $C^{2}$ over the open set $\mathcal{U}_{t}$ containing the LCS.

Proof: Since

$$
L(\mathbf{x}, t)= \pm\left\|\mathbf{x}-\mathbf{x}_{q}\right\|,
$$

we have

$$
\nabla L=\frac{ \pm 1}{\left\|\mathbf{x}-\mathbf{x}_{q}\right\|}\left\langle I-\frac{\mathrm{d} \mathbf{x}_{q}}{\mathrm{~d} \mathbf{x}}, \mathbf{x}-\mathbf{x}_{q}\right\rangle
$$

However, 


$$
\left\langle\frac{\mathrm{d} \mathbf{x}_{q}}{\mathrm{~d} \mathbf{x}}, \mathbf{x}-\mathbf{x}_{q}\right\rangle=0
$$

in $\mathcal{U}_{t}$ because the closest point on the LCS does not change with variations in the direction normal to $\mathcal{M}$. As a result,

$$
\nabla L=\frac{\mathbf{x}-\mathbf{x}_{q}}{ \pm\left\|\mathbf{x}-\mathbf{x}_{q}\right\|}=\frac{\mathbf{x}-\mathbf{x}_{q}}{L}=\hat{\mathbf{n}}\left(\mathbf{x}_{q}\right),
$$

where we have used the fact that $\mathbf{x}_{q}$ is the point on the LCS closest to $\mathbf{x}$; hence $\hat{\mathbf{n}}\left(\mathbf{x}_{q}\right)$ must be parallel to $\mathbf{x}-\mathbf{x}_{q}$. Recall that there is a unique $\mathbf{x}_{q}$ for each $\mathbf{x} \in \mathcal{U}_{t}$, by virtue of Theorem 3.1. By Corollary 2.1, the map $\hat{\mathbf{n}}\left(\mathbf{x}_{q}\right)$ that gives the normal vector at a point $\mathbf{x}_{q} \in \operatorname{LCS}$ is $C^{1}$; thus $\nabla L(\mathbf{x})$ $=\hat{\mathbf{n}}\left(\mathbf{x}_{q}\right)$ is $C^{1}$, which implies that $L$ is $C^{2}$.

The primary result that we derive is an estimate for the flux across a LCS in Theorem 3.3, which reduces to the result in Shadden et al. (2005) for planar systems. Below, $\hat{\mathbf{t}}$ denotes an arbitrary vector in the tangent plane to $\mathcal{M}$, i.e., $\langle\hat{\mathbf{t}}, \hat{\mathbf{n}}\rangle=0$. Let $\mathcal{L}$ be the Hessian of $L$ and note the following properties of $\mathcal{L}$ and $\Sigma$ :

Lemma 3.1: $\Sigma$ and $\mathcal{L}$ are self-adjoint.

Proof: The symmetry of $\Sigma$ is standard and is due to the symmetry of mixed partials. From $\Sigma(\mathbf{u}, \mathbf{v})=\Sigma(\mathbf{v}, \mathbf{u})$, we deduce that $\langle\mathbf{u}, \Sigma \mathbf{v}\rangle=\langle\mathbf{v}, \Sigma \mathbf{u}\rangle=\langle\Sigma \mathbf{u}, \mathbf{v}\rangle$ because the derivatives are necessarily real numbers. The same result applies to $\mathcal{L}$ since it is the (symmetric) Hessian of the scalar function $L$, which is $C^{2}$ by Theorem 3.2.

Lemma 3.2: For $L=0$ and for all $\hat{\mathbf{t}}$, we have

$$
\langle\hat{\mathbf{t}}, \hat{\mathbf{n}}\rangle=0 \Rightarrow\langle\hat{\mathbf{t}}, \Sigma \hat{\mathbf{n}}\rangle=\langle\hat{\mathbf{n}}, \Sigma \hat{\mathbf{t}}\rangle=0 .
$$

Proof: From Definition 2.1, LCS3 implies that $\nabla L=\hat{\mathbf{n}}$ is an eigenvector of $\Sigma$. Hence $\langle\hat{\mathbf{t}}, \Sigma \hat{\mathbf{n}}\rangle$ $=\lambda_{\min }(\Sigma)\langle\hat{\mathbf{t}}, \hat{\mathbf{n}}\rangle=0$, where $\lambda_{\text {min }}(\Sigma)$ is the smallest eigenvalue of $\Sigma$.

Corollary 3.2: For $L=0$ and an arbitrary vector $\mathbf{v}$, we have $\langle\hat{\mathbf{n}}, \Sigma \mathbf{v}\rangle=\langle\hat{\mathbf{n}}, \Sigma \hat{\mathbf{n}}\rangle\langle\hat{\mathbf{n}}, \mathbf{v}\rangle$.

Proof: Notice that any vector $\mathbf{v}$ can be written as

$$
\mathbf{v}=\hat{\mathbf{t}}+\langle\hat{\mathbf{n}}, \mathbf{v}\rangle \hat{\mathbf{n}}
$$

for a properly chosen $\hat{\mathbf{t}}$ in the tangent plane to $\mathcal{M}$. Computing $\langle\hat{\mathbf{n}}, \Sigma \mathbf{v}\rangle$ from the expansion in Eq. (13) and applying Lemma 3.2 gives the desired result.

Lemma 3.3: $\mathcal{L} \hat{\mathbf{n}}=0$ everywhere in $\mathcal{U}_{t}$.

Proof: Everywhere in $\mathcal{U}_{t}, L$ is $C^{2}$, so the gradient $\nabla L$ exists and is differentiable. In particular, $\|\nabla L\|=1$; therefore

$$
0=\nabla\left(\|\nabla L\|^{2}\right)=2 \mathcal{L} \nabla L=2 \mathcal{L} \hat{\mathbf{n}} .
$$

Lemma 3.4: On the LCS, i.e., for $L=0$,

$$
\langle\hat{\mathbf{n}}, \boldsymbol{\Sigma} \hat{\mathbf{n}}\rangle \frac{\partial L}{\partial t}=\frac{\partial\langle\hat{\mathbf{n}}, \nabla \sigma(\mathbf{x}, t ; T)\rangle}{\partial t} .
$$

Proof: Take $\mathbf{x}$ on the LCS at time $t$, i.e., $L(\mathbf{x}, t)=0$. Define $\mathbf{y}=\mathbf{x}+\alpha(\delta t) \hat{\mathbf{n}}$ such that $L(\mathbf{y}, t$ $+\delta t)=0$. In other words, $\mathbf{y}$ is at the intersection of the LCS at time $t+\delta t$ and the line starting at $\mathbf{x}$, orthogonal to the LCS at time $t$ (see Fig. 2). Since we require $\mathbf{y}=\mathbf{x}$ for $\delta t=0$, it follows that $\alpha(\delta t)$ is $\mathcal{O}(\delta t)$. Expanding $L$ to first order in $\delta t$ gives the following (where all derivatives on the right-hand side of the following equations are evaluated at $\mathbf{x}$ and $t$ unless otherwise specified): 


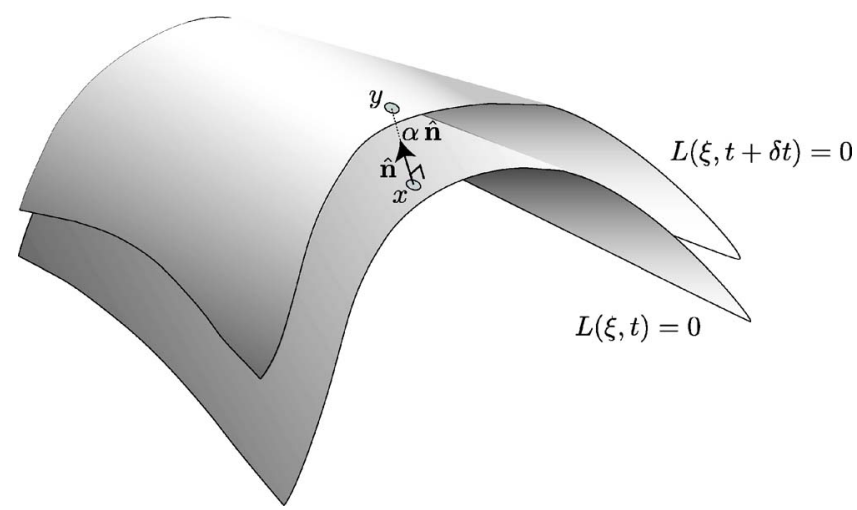

FIG. 2. Quantities discussed in Lemma 3.4.

$$
\begin{aligned}
0 & =L(\mathbf{y}, t+\delta t)=L(\mathbf{x}, t)+\alpha+\frac{\partial L}{\partial t} \delta t+\mathcal{O}\left(\delta t^{2}\right), \\
& =\alpha+\frac{\partial L}{\partial t} \delta t+\mathcal{O}\left(\delta t^{2}\right) .
\end{aligned}
$$

Therefore,

$$
\alpha=-\frac{\partial L}{\partial t} \delta t+\mathcal{O}\left(\delta t^{2}\right)
$$

Now expanding $\nabla L$ and plugging in Lemma 3.3 gives

$$
\left.\nabla L\right|_{\mathbf{y}, t+\delta t}=\nabla L+\frac{\partial \nabla L}{\partial t} \delta t+\mathcal{O}\left(\delta t^{2}\right) .
$$

Taylor expanding $\left.\nabla \sigma(\mathbf{x}, t ; T)\right|_{\mathbf{y}, t+\delta t}$ gives

$$
\left.\nabla \sigma(\mathbf{x}, t ; T)\right|_{\mathbf{y}, t+\delta t}=\nabla \sigma(\mathbf{x}, t ; T)+\alpha \boldsymbol{\Sigma} \hat{\mathbf{n}}+\frac{\partial \nabla \sigma(\mathbf{x}, t ; T)}{\partial t} \delta t+\mathcal{O}\left(\delta t^{2}\right) .
$$

From Eqs. (12) and (14) we have

$$
\left.\nabla \sigma(\mathbf{x}, t ; T)\right|_{\mathbf{y}, t+\delta t}=\nabla \sigma(\mathbf{x}, t ; T)-\frac{\partial L}{\partial t} \mathbf{\Sigma} \nabla L \delta t+\frac{\partial \nabla \sigma(\mathbf{x}, t ; T)}{\partial t} \delta t+\mathcal{O}\left(\delta t^{2}\right)
$$

Since $\mathbf{y}$ is on the LCS at time $t+\delta t$, we must have

$$
\begin{aligned}
0 & =\left\langle\left.\nabla L\right|_{\mathbf{y}, t+\delta t},\left.\quad \nabla \sigma(\mathbf{x}, t ; T)\right|_{\mathbf{y}, t+\delta t}\right\rangle \\
& =\langle\nabla L, \nabla \sigma(\mathbf{x}, t ; T)\rangle+\delta t\left[-\frac{\partial L}{\partial t}\langle\nabla L, \Sigma \nabla L\rangle+\frac{\partial\langle\nabla L, \nabla \sigma(\mathbf{x}, t ; T)\rangle}{\partial t}\right]+\mathcal{O}\left(\delta t^{2}\right) \\
& =\delta t\left[-\langle\hat{\mathbf{n}}, \boldsymbol{\Sigma} \hat{\mathbf{n}}\rangle \frac{\partial L}{\partial t}+\frac{\partial\langle\hat{\mathbf{n}}, \nabla \sigma(\mathbf{x}, t ; T)\rangle}{\partial t}\right]+\mathcal{O}\left(\delta t^{2}\right) .
\end{aligned}
$$

Hence, we get the desired result, since $\delta t$ is arbitrary.

By definition of the function $L$ it is not hard to show, as in Shadden et al. (2005), that 


$$
\frac{\mathrm{d} L}{\mathrm{~d} t}=\nabla L \cdot\left[\frac{\mathrm{d} \mathbf{x}}{\mathrm{d} t}-\frac{\mathrm{d} \mathbf{x}_{q}}{\mathrm{~d} t}\right] .
$$

On the LCS, i.e., for $L=0$, the two points $\mathbf{x}$ and $\mathbf{x}_{q}$ are equal; however, we think of $\mathbf{x}$ as being a Lagrangian or material point while $\mathbf{x}_{q}$ is viewed as a point that moves with the LCS. Therefore, the total flux across the LCS is given by

$$
\Phi(t)=\int_{\mathrm{LCS}} \frac{\mathrm{d} L}{\mathrm{~d} t} \mathrm{~d} s,
$$

where the integral is taken over the LCS, i.e., the codimension 1 surface $\mathcal{M}$. The next theorem contains an expression for $\mathrm{d} L / \mathrm{d} t$ based on quantities defining the FTLE and velocity fields.

Theorem 3.3: For $L=0$, we have

$$
\frac{\mathrm{d} L}{\mathrm{~d} t}=\frac{1}{\langle\hat{\mathbf{n}}, \boldsymbol{\Sigma} \hat{\mathbf{n}}\rangle}\left\langle\nabla \sigma(\mathbf{x}, t ; T), \frac{\partial \hat{\mathbf{n}}}{\partial t}-J \hat{\mathbf{n}}\right\rangle+\mathcal{O}(1 /|T|) .
$$

Proof: Lemma 3.4 gives

$$
\langle\hat{\mathbf{n}}, \boldsymbol{\Sigma} \hat{\mathbf{n}}\rangle \frac{\mathrm{d} L}{\mathrm{~d} t}=\frac{\partial\langle\hat{\mathbf{n}}, \nabla \sigma(\mathbf{x}, t ; T)\rangle}{\partial t}+\langle\hat{\mathbf{n}}, \boldsymbol{\Sigma} \hat{\mathbf{n}}\rangle\langle\hat{\mathbf{n}}, \mathbf{v}\rangle .
$$

Applying Corollary 3.2 and the chain rule for the derivative gives

$$
\langle\hat{\mathbf{n}}, \Sigma \hat{\mathbf{n}}\rangle \frac{\mathrm{d} L}{\mathrm{~d} t}=\left\langle\nabla \sigma(\mathbf{x}, t ; T), \frac{\partial \hat{\mathbf{n}}}{\partial t}\right\rangle+\left\langle\hat{\mathbf{n}}, \frac{\partial \nabla \sigma(\mathbf{x}, t ; T)}{\partial t}\right\rangle+\langle\hat{\mathbf{n}}, \Sigma \mathbf{v}\rangle .
$$

Using Corollary 3.1 in Eq. (17) gives Eq. (16).

The above theorem provides an estimate for the instantaneous flux across a LCS. For twodimensional systems, the estimate given in Eq. (16) is nearly identical to the expression in Shadden et al. (2005) for the case of two-dimensional dynamical systems. In particular, we note that the flux is inversely proportional to how well defined the LCS is, which is measured by the term $\left\langle\hat{\mathbf{n}}, \sum \hat{\mathbf{n}}\right\rangle$. The term

$$
\left\langle\nabla \sigma(\mathbf{x}, t ; T), \frac{\partial \hat{\mathbf{n}}}{\partial t}-J \hat{\mathbf{n}}\right\rangle
$$

can be thought of as the difference between the local rotation rate of the LCS and the instantaneous rotation due to the Eulerian velocity field measured in the direction of $\nabla \sigma$. The $\mathcal{O}(1 /|T|)$ term in Eq. (16) decreases rapidly with the integration time used to compute the FTLE field.

Theorem 3.3 does not involve the tangent vector $\hat{\mathbf{t}}$. This property suggests that the flux through a LCS is determined by the FTLE in its normal bundle. More specifically, $\partial \hat{\mathbf{n}} / \partial t, J \hat{\mathbf{n}}$, and $\left\langle\hat{\mathbf{n}}, \sum \hat{\mathbf{n}}\right\rangle$ are, respectively, the rotation rate of the normal vector, the velocity gradient in the normal direction, and the curvature in the normal direction. In Theorem 3.3, the only term related to the tangent bundle is $\nabla \sigma$, since $\hat{\mathbf{n}} \cdot \nabla \sigma=0$ by definition. Nevertheless, $\sigma$ characterizes the ejection rate (or attraction rate for $T<0$ ) in a direction transverse to the LCS. As a result, the term $\nabla \sigma$ does not depend on the dynamics in the tangent bundle. It is, instead, a measure of how homogeneous the normal attraction or repulsion is along the LCS.

\section{EXAMPLE: THREE-DIMENSIONAL RAYLEIGH-BÉNARD CONVECTION CELL}

In this section, we illustrate the computation of LCS on a three-dimensional dynamical model of time-dependent Rayleigh-Bénard convection derived from the work of Solomon and Gollub (1988, 1990, 1991). 


\section{A. The model}

The model of Solomon and Gollub involves an infinite array of two-dimensional convection cells bounded from above and below by horizontal free-slip boundaries. It is derived from the stream function

$$
\psi=\frac{A}{k} \sin [k[x-g(t)]] \sin z,
$$

where $A$ is the maximum vertical velocity in the system, $k$ is the wave number, and $g(t)$ dictates the lateral motion of the cells. This kinematic model does not satisfy the Navier-Stokes equations, but as in Solomon and Gollub (1988), the parameters are chosen to match flow patterns observed during experiments. The methods of this paper are, however, independent of the source of the velocity field. One can compute LCS for a kinematic model (as in the present example), for an analytic model that satisfies the Navier-Stokes equations, for the output of a CFD program, or from empirical data.

To extend this model to three-dimensional cylindrical cells, we start by considering the autonomous version of Solomon and Gollub's model [i.e., $g(t)=0$ ] applied to the vertical, $z$, and the radial, $r=\sqrt{x^{2}+y^{2}}$, variables. In cylindrical coordinates, the stream function is $\Psi=r^{2} \psi(r, z)$ and the vector field is given by $((1 / r)(\partial \Psi / \partial z),-(1 / r)(\partial \Psi / \partial r))$. Converting to Cartesian coordinates we obtain

$$
\begin{gathered}
\dot{x}=\frac{A}{k} x \sin k r \cos z, \\
\dot{y}=\frac{A}{k} y \sin k r \cos z, \\
\dot{z}=-A \sin z\left[r \cos k r+\frac{2}{k} \sin k r\right],
\end{gathered}
$$

where $r^{2}=x^{2}+y^{2}$.

Notice from system (18) that the vector field vanishes when $r=0$. This could represent a case where there is a thin wire placed along the axis, which has a no-slip boundary condition. This condition will have little consequence on the transport structure of the time-dependent system since it is nonhyperbolic and of codimension 2.

The vector field is defined for all of $\mathbb{R}^{3}$ and is spatially periodic in the radial and axial directions. In the radial direction there is an array of concentric cells. The flow field for the innermost cell, which is the cell considered here, is shown in Fig. 3. The radius and diameter of the cell are both $\pi / 2$. For the steady system, Eq. (18), the cell is invariant and all the flow is confined to the region shown. In particular, the circumference of the cell is bounded by a family of heteroclinic trajectories. Next, we show that if a time-dependent forcing is added to the system, these heteroclinic connections break, resulting in transport to and from this center cell, which is captured by the LCS.

To add unsteadiness to the model, we follow the same procedure as in Solomon and Gollub (1988). We replace $x$ in the autonomous velocity field by $\xi=x-g(t)$. Such a spatially homogeneous, time-dependent translation does not affect the divergence. ${ }^{4}$

The resulting model is

\footnotetext{
${ }^{4}$ Any autonomous, divergence-free vector field $\dot{\mathbf{x}}=f(\mathbf{x})$ can be transformed into a divergence-free, time-dependent system $\dot{\mathbf{x}}=f(\mathbf{x}-\mathbf{p}(t))$ for any $\mathbf{p}(t)$. For the Rayleigh-Bénard cell, the translation $\mathbf{p}(t)$ is chosen to align with the $x$ axis.
} 


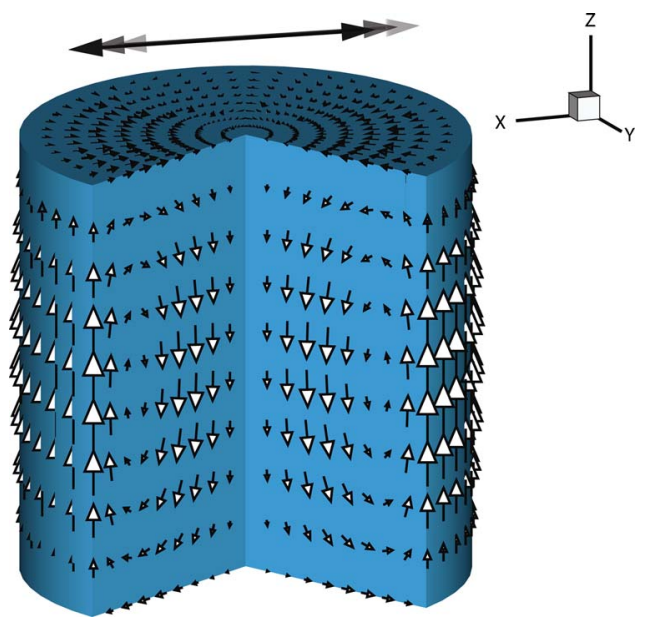

FIG. 3. Sectioned model of Rayleigh-Bénard convection. The flow is made time dependent by shifting the cell back and forth in the $x$ direction, as indicated.

$$
\begin{gathered}
\dot{x}=\frac{A}{k} \xi \sin k \rho \cos z, \\
\dot{y}=\frac{A}{k} y \sin k \rho \cos z, \\
\dot{z}=-A \sin z\left(\rho \cos k \rho+\frac{2}{k} \sin k \rho\right),
\end{gathered}
$$

where $\xi=x-g(t)$ and $\rho^{2}=\xi^{2}+y^{2}$.

\section{B. Three-dimensional Lagrangian coherent structures}

The LCS for the model given in Eq. (19) are shown in Fig. 4 for parameters $A=0.24$ and $k=2$. The red and blue surfaces are the repelling and attracting LCS, respectively. The integration time used to compute the FTLE fields was $T=12$. In general, longer integration times will allow the LCS to be better resolved but at the price of a higher computational cost.

To compute the LCS, a specific forcing $g(t)$ had to be chosen. Since the main advantage of the method developed in this paper is to accommodate arbitrary time dependence, selecting a periodic or even quasiperiodic forcing would not explore the full potential of LCS computation. To get a random forcing, $g(t)$ is chosen as a realization of a Gaussian noise with unit variance and unit correlation in time. Such a one-dimensional displacement can be easily computed by taking the inverse Fourier transform of the Fourier transform of a random sequence fitted to the desired Gaussian spectrum [Lekien et al. (2005a)]. Notice that we do not select $g(t)$ randomly at each time step $t$; this would result in a nonsmooth system. Furthermore, we do not investigate statistical properties of the system. The function $g(t)$ is a realization of the random process but, once computed, it becomes a deterministic oscillating forcing.

To avoid the lateral motion of the image, the LCS in Fig. 4 are shown in the $(\xi, y, z)$ coordinate system, instead of $(x, y, z)$. High resolution snapshots and complete animations can be downloaded at http://www.lekien.com/ francois/papers/lcs $3 \mathrm{~d}$.

\section{Dynamics and transport}

Figures 4 and 5 reveal LCS that bound the inner cell. For the time-independent system $(g(t)$ constant), the attracting and repelling LCS coincide and no transport to or from the cell would be present. For the randomly chosen $g(t)$, the LCS intersect to form sequences of lobes. Fluid is 

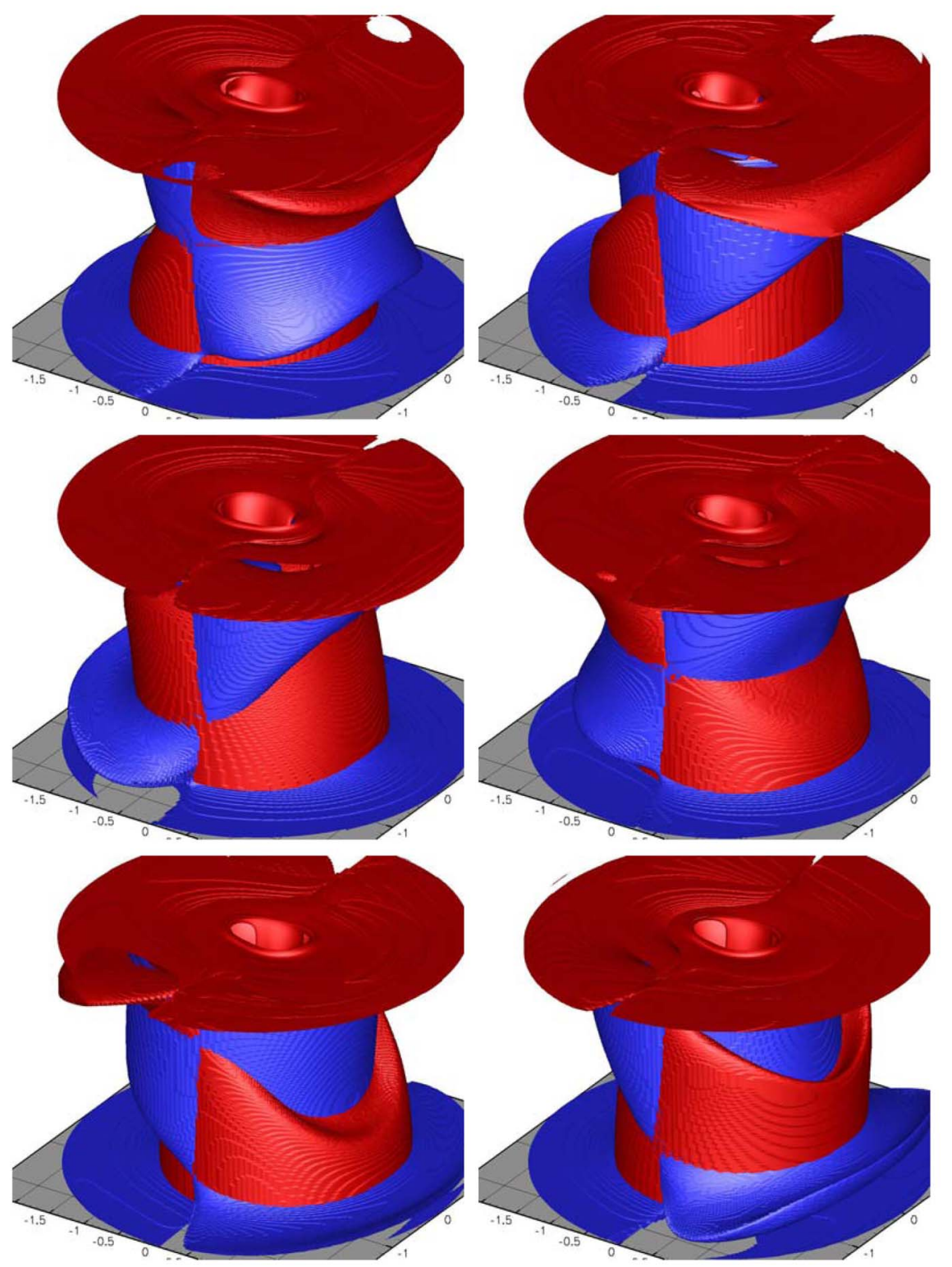

FIG. 4. Attracting (blue) and repelling (red) LCS for a model of Rayleigh-Bénard convection. The center cell is bound by the moving LCS. The intersections between the LCS lead to lobes whose dynamics govern transport to and from the cell.

entrained and detrained as these three-dimensional lobes deform from lobes of fluid outside the cell into lobes of fluid inside the cell, and vice versa. Such lobe mechanics have been routinely shown for near-integrable or near-periodic systems in two-dimensional examples [e.g., Guckenheimer and Holmes (1986), Rom-Kedar (1990), Rom-Kedar and Wiggins (1991), Beigie et al. (1991), and Coulliette and Wiggins (2000)] and even for three-dimensional computations [e.g., Beigie (1995) and Wysham and Meiss (2006)]. However, the significance of the method presented here is to generalize these notions to systems with arbitrary time dependence.

It is interesting to note that lobes formed by LCS are not always as regular as the ones presented in this paper. In fact, lobes need not exist at all. Beigie (1995) presents examples where lobes in three-dimensional systems can have connections between the lobes. In some cases, none 

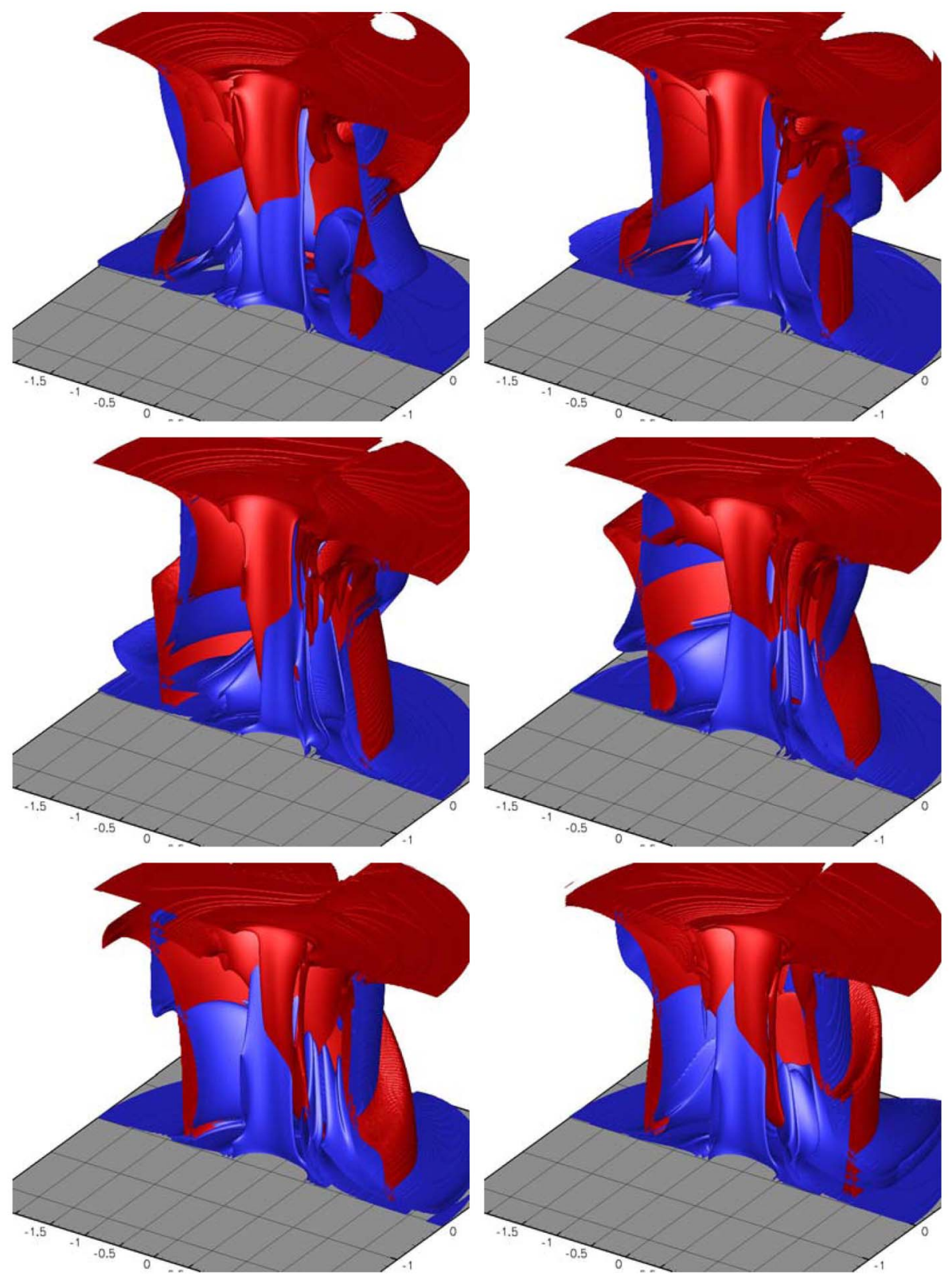

FIG. 5. Attracting (blue) and repelling (red) LCS for a model of Rayleigh-Bénard convection. The vertical section reveals the complex entanglement of the lobes inside the center cell. Transport of particles can be derived from the shape and dynamics of the lobes.

of the lobes are disjoint from the others and it is impossible to describe transport in terms of lobe mapping. In such systems, one cannot identify localized lobes carrying fluid from one region to another. Nevertheless, for the particular model of Rayleigh-Bénard convection used in this paper, one can prove formally that regular sequences of disjoint lobes govern transport (see the Appendix).

Figure 5 shows the inside of the separatrix and reveals highly convoluted lobes. In particular, lobes have secondary intersections, which explains how complex, chaotic dynamics can take place in such a structured system [Rom-Kedar (1990)]. Since the goal of the definition in this paper is 


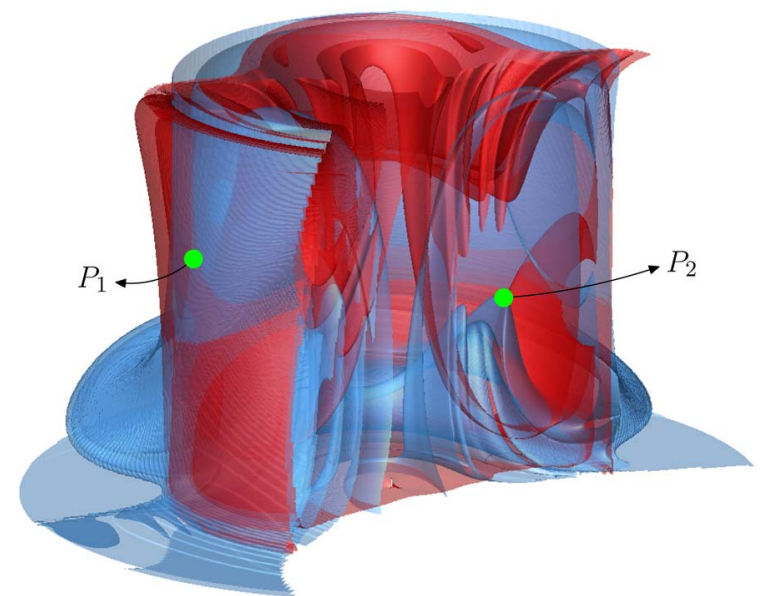

FIG. 6. Points where flux is evaluated. $P_{1}$ is located on the initial segment of the repelling LCS and $P_{2}$ on the tip of the first lobe of the attracting LCS.

to find hypersurfaces that locally maximize the Lyapunov exponent, it is evident that the system is sensitive to initial conditions in a neighborhood of the LCS. Although there does not exist any formal theorem to support this assertion, it should be clear from Fig. 5 that a chaotic Cantor set is expected to be found in the entanglement of the two LCS. Smale and Morse proved the existence of chaos near the hyperbolic manifolds of two-dimensional periodic systems [Smale (1965); Guckenheimer and Holmes (1986)]. The extension to chaotic forcing and higher dimensional systems is work in progress, but these computations of LCS reveal that such chaotic motion is the general behavior in the vicinity of intersecting LCS, even for systems that are of higher dimension or more complex time dependency than previously studied.

\section{Flux across Lagrangian coherent structures}

Theorem 3.3 provides a convenient way to find an upper bound on the flux of particles across the LCS. The instantaneous flux is given by

$$
\frac{\mathrm{d} L}{\mathrm{~d} t}=\frac{\langle\nabla \sigma, \partial \hat{\mathbf{n}} / \partial t-J \hat{\mathbf{n}}\rangle}{\langle\hat{\mathbf{n}}, \Sigma \hat{\mathbf{n}}\rangle} \leqslant \frac{\|\nabla \sigma\|}{(\hat{\mathbf{n}}, \Sigma \hat{\mathbf{n}})}\left(\left\|\frac{\partial \hat{\mathbf{n}}}{\partial t}\right\|+\|J \hat{\mathbf{n}}\|\right)
$$

The last term in the equation above is the hardest to evaluate. Nevertheless, notice that each component in $J$ is bounded by $4 A / k \approx 0.5$. We evaluate the other terms for two points $P_{1}$ and $P_{2}$ indicated in Fig. 6. $P_{1}$ is on the main sheet of the LCS (before intersections create lobes) and $P_{2}$ is located at the tip the first internal lobe.

\begin{tabular}{lcrlccccc} 
& \multicolumn{1}{c}{$y$} & \multicolumn{1}{c}{$y$} & \multicolumn{1}{c}{$z$} & \multicolumn{1}{c}{$\|\nabla \sigma\|$} & $\langle\hat{\mathbf{n}}, \Sigma \hat{\mathbf{n}}\rangle$ & $\|\partial \hat{\mathbf{n}} / \partial t\|$ & $\|J \hat{\mathbf{n}}\|$ & $|\mathrm{d} L / \mathrm{d} t|$ \\
\hline Point $P_{1}$ & 0.000 & -1.5225 & 2.0 & 0.005142 & 11194.6 & $\leqslant 10^{-4}$ & $\leqslant 0.5$ & $\leqslant 2.3 \times 10^{-7}$ \\
Point $P_{2}$ & 0.000 & 1.0147 & 0.334 & 0.012614 & 5154.0 & $\leqslant 10^{-2}$ & $\leqslant 0.5$ & $\leqslant 1.2 \times 10^{-6}$
\end{tabular}

As in Shadden et al. (2005), it is convenient to compare this flux estimate to the typical magnitude of the velocity field. For both points $P_{1}$ and $P_{2}$, the velocity ranges around $\|\mathbf{v}\| \approx 0.2$. Furthermore, the maximum velocity inside the central cell (i.e., $\xi^{2}+y^{2}<\pi^{2}$ ) is given by $\sqrt{2\left(A^{2} \pi^{2} / k^{2}\right)+A^{2}} \approx 0.6$. The flux, as computed above, is therefore several orders of magnitude below the usual velocity. Also notice that the upper bound $\|J \hat{\mathbf{n}}\|<4 A / k$ is highly conservative. Computing this explicitly could lower the flux estimate significantly.

The estimate given in Theorem 3.3 is an instantaneous flux. On one hand a negligible instan- 
taneous flux can aggregate to a non-negligible integrated flux or, on the other hand, the flux can rapidly change sign and thus remain negligible. Experience has shown that the net flux of Lagrangian particles across a LCS is usually much lower than what Theorem 3.3 indicates and LCS can be considered as material lines except at times when the LCS are very poorly defined. In fact, the flux that we have computed is on the same order of magnitude as the numerical error of computations of trajectories. That is, by computing a true invariant manifold, one would not normally achieve a smaller flux than what is observed by defining the LCS from FTLE.

We would like to point out that LCS can become poorly defined when the flow structure undergoes a large qualitative change. This is common for aperiodic systems where the flow drastically changes regimes. In this case a LCS that was well defined may become poorly defined and diminishes in length as time passes. For such a LCS, the curvature $\langle\hat{\mathbf{n}}, \Sigma \hat{\mathbf{n}}\rangle$ becomes less pronounced and thus, according to Theorem 3.3, the LCS loses its impermeability. However, this is consistent with the realization that the flow structure is changing regimes and the LCS, which may no longer be relevant, is naturally adjusting to the regime change of the flow. Therefore, it is not necessarily a weakness that LCS are not always perfectly Lagrangian. Theorem 3.3 allows us to track the relevancy of the LCS.

\section{CONCLUSIONS}

This paper extends the ideas and proofs presented in Shadden et al. (2005) to $n$-dimensional systems. Here, LCS are defined as codimension 1 maximizing surfaces of the FTLE field, which is made precise in Definition 2.1. It is shown that the advection property of the LCS shown in Shadden et al. (2005) can be extended to higher dimension systems. These results are applied to a model of Rayleigh-Bénard convection based on the work of Solomon and Gollub.

Previous dynamical systems methods for unsteady systems have mostly been applied to twodimensional problems. However, a vast majority of interesting unsteady fluid systems are inherently three dimensional. The method presented here has no inherent limitation imposed by the dimension of the system. However, the computational cost increases exponentially with the dimension of the system based on current computation techniques used to produce the results shown here. Therefore, to ensure that the method presented in this paper can be used as a practical tool in the analysis of unsteady systems with dimension higher than 2, it is important to develop efficient numerical methods. This is currently an active area of research. Additionally, visualization of LCS becomes difficult for higher dimensional systems.

\section{ACKNOWLEDGMENTS}

This work was partially supported by Office of Naval Research Grant No. N00014-04-1-0534 and has profited from the motivation and support of the entire Adaptive Sampling and Prediction (ASAP) team. The authors are grateful to Naomi Leonard for her valuable support and thank Chad Coulliette, George Haller, Manuel Fiadeiro, and Thomas Curtin for helpful remarks and enlightening discussions.

\section{APPENDIX: THREE-DIMENSIONAL LOBES}

Transport in the three-dimensional model given in Eq. (19) is governed by two sequences of lobes. Fluid inside the lobes will be progressively transported from one side of the center cell to the other. Furthermore, this is the only mechanism of transport to and from the cell.

Such transport mechanisms are the general rules near hyperbolic tangles in two-dimensional systems [see, e.g., Smale (1965) and Rom-Kedar (1990)]. In higher dimensions, the geometry might be more complex. In particular, the lobes might not be disjoint and do not lead to the same transport mechanism [Beigie (1995)].

In the case of the Rayleigh-Bénard convection in this paper, the resulting lobes are, however, always disjoint. We can show this property by noticing that the three-dimensional model in Eq. (19) can be reduced to a bundle of two-dimensional invariant planes. Indeed, the intersection of the two planes $x=g(t)$ and $y=0$ defines a line that is parallel to the $z$ axis and intersects the $x$ axis 
at $x=g(t)$. The equation $y=K \xi$, where $K \in \mathbb{R}$, parametrizes all planes that contain this line. Each of these vertical planes remains invariant. To see this, let $\tilde{\theta}$ denote the angle the plane $y=K \xi$ makes with the $x$ axis. Thus we have

$$
\begin{aligned}
& \xi=\rho \cos \tilde{\theta}, \\
& y=\rho \sin \tilde{\theta} .
\end{aligned}
$$

The vector $(\sin \tilde{\theta},-\cos \tilde{\theta})$ is orthogonal to the plane $y=K \xi$. Using Eq. (A1), we can show

$$
[\sin \tilde{\theta}-\cos \tilde{\theta}]\left[\begin{array}{l}
\dot{\xi} \\
\dot{y}
\end{array}\right]=-\rho \dot{\tilde{\theta}}
$$

However, it is easy to show that $\dot{\tilde{\theta}}=0$ for all $\rho \neq 0$. Hence the "moving" planes $y=K \xi$ are invariant. To take advantage of the reduction, we can fix $\tilde{\theta}=\tilde{\theta}_{0}$ for each plane and allow negative values of $\rho$ to avoid the discontinuity of $\tilde{\theta}$ along $\rho=0$. The dynamics on each plane is given by

$$
\begin{gathered}
\dot{\rho}=\frac{A}{k} \rho \sin k \rho \cos z-g^{\prime}(t) \cos \tilde{\theta}_{0}, \\
\dot{z}=-A \sin z\left[\rho \cos k \rho+\frac{2}{\kappa} \sin k \rho\right] .
\end{gathered}
$$

This form results from a nonlinear coordinate change from the physical plane $(x, y, z)$ to the coordinate system $(\rho, z, \bar{\theta})$, where $\dot{\bar{\theta}}=0$. One can also see this transformation as a mapping from the cylindrical coordinates $(r, z, \theta)$ to the coordinates $(r, z, \bar{\theta})$. This transformation is also nonlinear and nontrivial since $\bar{\theta}=\sin ^{-1}(r \sin \theta / \rho)$ is highly nonlinear. Indeed, $r$ depends on $\rho$ as

$$
r=g(t) \cos \theta+\sqrt{\rho^{2}-g^{2}(t) \sin ^{2} \theta}
$$

hence the full transformation reads

$$
\bar{\theta}=\sin ^{-1}\left(\frac{[g(t) / 2] \sin 2 \theta+\sin \theta \sqrt{\rho^{2}-g^{2}(t) \sin ^{2} \theta}}{\rho}\right),
$$

which is nonlinear for $g(t) \neq 0$.

To study transport and lobe geometry, one can restrict the analysis to an invariant moving plane. Since the plane is two dimensional, transport is governed by sequences of two-dimensional lobes. The three-dimensional lobes that we observe in the full system are the stacking of the two-dimensional lobes of each invariant plane. These lobes will not be disjoint, as in Beigie (1995), because that would imply motion of the particles in a direction transverse to the invariant planes.

${ }^{1}$ Arratia, P. E., Voth, G. A., and Gollub, J. P., "Stretching and mixing of non-Newtonian fluids in time-periodic flows," Phys. Fluids 17, 053102 (2005).

${ }^{2}$ Beigie, D., "Multiple separatrix crossing in multi-degree-of-freedom Hamiltonian flows," J. Nonlinear Sci. 5, 57-103 (1995).

${ }^{3}$ Beigie, D., Leonard, A., and Wiggins, S., "A global study of enhanced stretching and diffusion in chaotic tangles," Phys. Fluids A 3, 1039-1050 (1991).

${ }^{4}$ Coulliette, C., Lekien, F., Haller, G., Paduan, J., and Marsden, J. E., "Optimal pollution mitigation in Monterey Bay based on coastal radar data and nonlinear dynamics," Environ. Eng. Sci. (to be published).

${ }^{5}$ Coulliette, C. and Wiggins, S., "Intergyre transport in a wind-driven, quasigeostrophic double gyre: An application of lobe dynamics," Nonlinear Processes Geophys. 7, 59-85 (2000).

${ }^{6}$ Dieci, L. and Eirola, T., "On smooth decompositions of matrices," SIAM J. Matrix Anal. Appl. 20, 800-819 (1999). 
${ }^{7}$ Green, M. A., Rowley, C. W., and Haller, G., "Detection of Lagrangian coherent structures in three-dimensional turbulence,” J. Fluid Mech. 572, 111-120 (2007).

${ }^{8}$ Guckenheimer, J. and Holmes, P., Nonlinear Oscillations, Dynamical Systems, and Bifurcations of Vector Fields (Springer-Verlag, New York, 1986).

${ }^{9}$ Haller, G., "Distinguished material surfaces and coherent structures in three-dimensional fluid flows," Physica D 149, 248-277 (2001).

${ }^{10}$ Haller, G., "Finding finite-time invariant manifolds in two-dimensional velocity fields," Chaos 10, 99-108 (2000).

${ }^{11}$ Haller, G., "Lagrangian coherent structures from approximate velocity data," Phys. Fluids 14, 1851-1861 (2002).

${ }^{12}$ Haller, G. and Poje, A. C., "Finite time transport in aperiodic flows," Physica D 119, 352-380 (1998).

${ }^{13}$ Hirsch, M. W. and Smale, S., Differential Equations, Dynamical Systems and Linear Algebra (Academic, New York, 1974).

${ }^{14}$ Homburg, A. J., De Vilder, R., and Sands, D., "Computing invariant sets," Int. J. Bifurcation Chaos Appl. Sci. Eng. 13, 497-504 (2003).

${ }^{15}$ Inanc, T., Shadden, S. C., and Marsden, J., Proceedings of the 2005 American Control Conference, Portland, OR, 2005, pp. 674-679 (http://ieeexplore.ieee.org/xpls/abs_all.jsp?arnumber=1470035).

${ }^{16}$ Jones, C. K. R. T. and Winkler, S., Handbook of Dynamical Systems, edited by B. Fiedler (Elsevier, Amsterdam, 2002), Vol. 2, pp. 55-92.

${ }^{17}$ Joseph, B. and Legras, B., "Relation between kinematic boundaries, stirring, and barriers for the Antarctic polar vortex," J. Atmos. Sci. 59, 1198-1212 (2002).

${ }^{18}$ Kato, T., Perturbation Theory for Linear Operators (Classics in Mathematics) (Springer-Verlag, Berlin, 1995).

${ }^{19}$ Koh, T. Y. and Legras, B., "Hyperbolic lines and the stratospheric polar vortex," Chaos 12, 382-394 (2002).

${ }^{20}$ Krauskopf, B., Osinga, H. M., Doedel, E. J., Henderson, M. E., Guckenheimer, J. M., Vladimirsky, A., Dellnitz, M., and Junge, O., "A survey of methods for computing (un)stable manifolds of vector fields," Int. J. Bifurcation Chaos Appl. Sci. Eng. 15, 763-791 (2005).

${ }^{21}$ Lekien, F., Berman, S., and Fiorelli, E., Multivariate Gaussian noise generator, 2005a (http://www.lekien.com/ francois/ software/rndfieldgen).

${ }^{22}$ Lekien, F., Coulliette, C., Bank, R., and Marsden, J., "Open-boundary modal analysis: Interpolation, extrapolation, and filtering," J. Geophys. Res., [Oceans] 109, C12004 (2004).

${ }^{23}$ Lekien, F., Coulliette, C., Mariano, A. J., Ryan, E. H., Shay, L. K., Haller, G., and Marsden, J., "Pollution release tied to invariant manifolds: A case study for the coast of Florida," Physica D 210, 1-20 (2005b).

${ }^{24}$ Lekien, F. and Leonard, N. E., "Dynamically consistent Lagrangian coherent structures," Experimental Chaos: 8th Experimental Chaos Conference, AIP Conf. Proc. 742, pp. 132-139 (2004).

${ }^{25}$ Lekien, F. and Marsden, J., "Tricubic interpolation in three dimensions," Int. J. Numer. Methods Eng. 63, 455-471 (2005).

${ }^{26}$ Mancho, A. M., Small, D., and Wiggins, S., "Computation of hyperbolic trajectories and their stable and unstable manifolds for oceanographic flows represented as data sets," Nonlinear Processes Geophys. 11, 17-33 (2004).

${ }^{27}$ Ottino, J. M., The Kinematics of Mixing: Stretching, Chaos, and Transport (Cambridge University Press, New York, 1989).

${ }^{28}$ Padberg, K., Ph.D. thesis, University of Paderborn, 2005.

${ }^{29}$ Pierrehumbert, R. T., "Large-scale horizontal mixing in planetary-atmospheres," Phys. Fluids A 3, 1250-1260 (1991).

${ }^{30}$ Pierrehumbert, R. T. and Yang, H., "Global chaotic mixing on isentropic surfaces," J. Atmos. Sci. 50, 2462-2480 (1993).

${ }^{31}$ Rom-Kedar, V., "Transport rates of a class of 2-dimensional maps and flows," Physica D 43, 229-268 (1990).

${ }^{32}$ Rom-Kedar, V., Leonard, A., and Wiggins, S., "An analytical study of transport, mixing and chaos in an unsteady vortical flow," J. Fluid Mech. 214, 347-394 (1990).

${ }^{33}$ Rom-Kedar, V. and Wiggins, S., "Transport in 2-dimensional maps: Concepts, examples, and a comparison of the theory of Rom-Kedar and Wiggins with the Markov model of Mackay, Meiss, Ott, and Percival," Physica D 51, 248-266 (1991).

${ }^{34}$ Shadden, S. C., Dabiri, J. O., and Marsden, J. E., "Lagrangian analysis of fluid transport in empirical vortex rings," Phys. Fluids 18, 047105 (2006).

${ }^{35}$ Shadden, S. C., Lekien, F., and Marsden, J. E., "Definition and properties of Lagrangian coherent structures from finite-time Lyapunov exponents in two-dimensional aperiodic flows," Physica D 212, 271-304 (2005).

${ }^{36}$ Smale, S., in Differential and Combinatorial Topology, edited by S. S. Cairns (Princeton University Press, Princeton, NJ, 1965).

${ }^{37}$ Solomon, T. H. and Gollub, J. P., "Passive transport in steady Rayleigh-Bénard convection," Phys. Fluids 31, 1372-1379 (1988).

${ }^{38}$ Solomon, T. H. and Gollub, J. P., "Sheared boundary-layers in turbulent Rayleigh-Bénard convection," Phys. Rev. Lett. 64, 2382-2385 (1990).

${ }^{39}$ Solomon, T. H. and Gollub, J. P., "Thermal-boundary layers and heat-flux in turbulent convection: The role of recirculating flows," Phys. Rev. A 43, 6683-6693 (1991).

${ }^{40}$ Toner, M. and Poje, A. C., "Lagrangian velocity statistics of directed launch strategies in a Gulf of Mexico model," Phys. Rev. A 43, 6683-6693 (2004).

${ }^{41}$ Voth, G. A., Haller, G., and Gollub, J. P., "Experimental measurements of stretching fields in fluid mixing," Phys. Rev. Lett. 88, 254501 (2002).

${ }^{42}$ Wysham, D. B. and Meiss, J. D., "Iterative techniques for computing the linearized manifolds of quasiperiodic tori," Chaos 16, 023129 (2006) 